\title{
Relativistic terahertz radiation generated by direct-laser-accelerated electrons from laser-foil interactions
}

\author{
$\mathrm{Ke} \mathrm{Hu} \odot$ and Longqing $\mathrm{Yi} \odot^{*}$ \\ Department of Physics, Chalmers University of Technology, 41296 Gothenburg, Sweden
}

(Received 19 March 2020; revised 15 June 2020; accepted 7 August 2020; published 26 August 2020)

\begin{abstract}
A scheme for generating powerful terahertz $(\mathrm{THz})$ radiation based on laser-solid interactions is proposed. When a $p$-polarized femtosecond laser impinges obliquely on a plane solid target and the target partially blocks the laser energy, surface electrons are extracted to the vacuum and accelerated by the laser fields, forming a low-divergence electron beam. A half-cycle $\mathrm{THz}$ radiation pulse is emitted simultaneously as the beam passes by the edge of the target, due to a mechanism similar to coherent transition radiation. Our particle-in-cell simulations show that a relativistic $\mathrm{THz}$ pulse can be obtained with an energy of a few tens of millijoule and a conversion efficiency above $1 \%$, with existing joule level femtosecond laser sources.
\end{abstract}

DOI: 10.1103/PhysRevA.102.023530

\section{INTRODUCTION}

Powerful terahertz radiation sources have attracted considerable attention due to their applications in many fields of science [1-3], such as THz spectroscopy of condensed matter or biological issues [4-6], nonlinear THz optics [7], and resonant control of materials [8,9]. Conventional laser-based $\mathrm{THz}$ radiation sources include optical rectification from nonlinear crystals [10-12], two-color laser filamentation [13,14], and switched photoconducting antennnas [15]. The peak fields are limited to the order of a few $\mathrm{MV} / \mathrm{cm}$, and the radiation energies are smaller than $1 \mathrm{~mJ}$. Another option, accelerator driven sources, can produce $\mathrm{THz}$ radiation with electromagnetic fields over $10 \mathrm{MV} / \mathrm{cm}$ and energies over $600 \mu \mathrm{J}$ [16]. However, they require linacs or storage rings to accelerate ultrashort relativistic electron bunches. The low accessibility of such large-scale, expensive facilities hinders the broad research on this approach.

Recently, relativistic laser-plasma interactions have been considered as a new method to produce strong $\mathrm{THz}$ radiation $[17,18]$. When a solid foil is irradiated by a pump laser with intensities over $10^{18} \mathrm{~W} / \mathrm{cm}^{2}$, produced fast electrons pass through the front and rear surfaces of the target, which lead to coherent transition radiation (CTR) of THz pulses in backward and forward directions, respectively [19,20]. Several experimental and numerical studies have shown that the energy of fast electrons is normally on the order of $\sim 100 \mathrm{keV}$ or $\sim \mathrm{MeV}$ [21-23], so most of the hot electrons in the low-energy end of the spectrum do not contribute to $\mathrm{THz}$ generation, as

\footnotetext{
*longqing@chalmers.se
}

Published by the American Physical Society under the terms of the Creative Commons Attribution 4.0 International license. Further distribution of this work must maintain attribution to the author(s) and the published article's title, journal citation, and DOI. Funded by Bibsam. they cannot escape the electrostatic fields near the target bulk. Besides, a large beam divergence $\left(\sim 40^{\circ}\right)$ is typically observed in the experiments [24], which significantly suppresses conversion efficiency [20]. The strongest $\mathrm{THz}$ radiation reported in laser-foil experiments is multimillijoule [25]. However, the total conversion efficiency is on the order of $10^{-3}$.

Laser-driven electron emission from nanostructured plasmas is a promising method for producing more collimated electron beams. These nanostructured plasmas include nanotips $[26,27]$ or nanoclusters [28-30], with sizes smaller than the laser wavelength. The electrons are first emitted within one laser cycle and then accelerated by the laser field based on the mechanism of vacuum laser acceleration (VLA). Recent results show that a $100 \mathrm{pC}$, few $\mathrm{MeV}$ electron beam can be obtained with a $10^{20} \mathrm{~W} / \mathrm{cm}^{2}$ few-cycle laser pulse [31].

In this paper, we propose a scheme that leverages this recent progress in VLA to generate powerful $\mathrm{THz}$ radiation. When a pump laser impinges obliquely on a solid foil, and the target partially blocks the laser, as only the edge of the foil interacts with the laser pulse, well-collimated surface electrons are produced similar to those generated in nanostructured plasmas. When such an electron beam is accelerated away from the target, it produces a CTR-like radiation due to the optical inhomogeneities in space. It should be noted that the radiation in question shares several important features with CTR. In particular, the radiation is coherent for wavelength longer than the duration of the electron beam, and radiated power greatly benefits from a small beam divergence. Nevertheless, it is not a typical CTR, since the properties of the particle beam are dramatically modified by VLA in the vicinity of the target (within the formation length [20] of CTR); thereby the dynamics following the transmission through the plasma-vacuum interface has a profound impact on the radiation field. Due to the complexity of the problem, fully kinetic particle-in-cell (PIC) simulations are employed to study the radiation properties and the electron dynamics. 

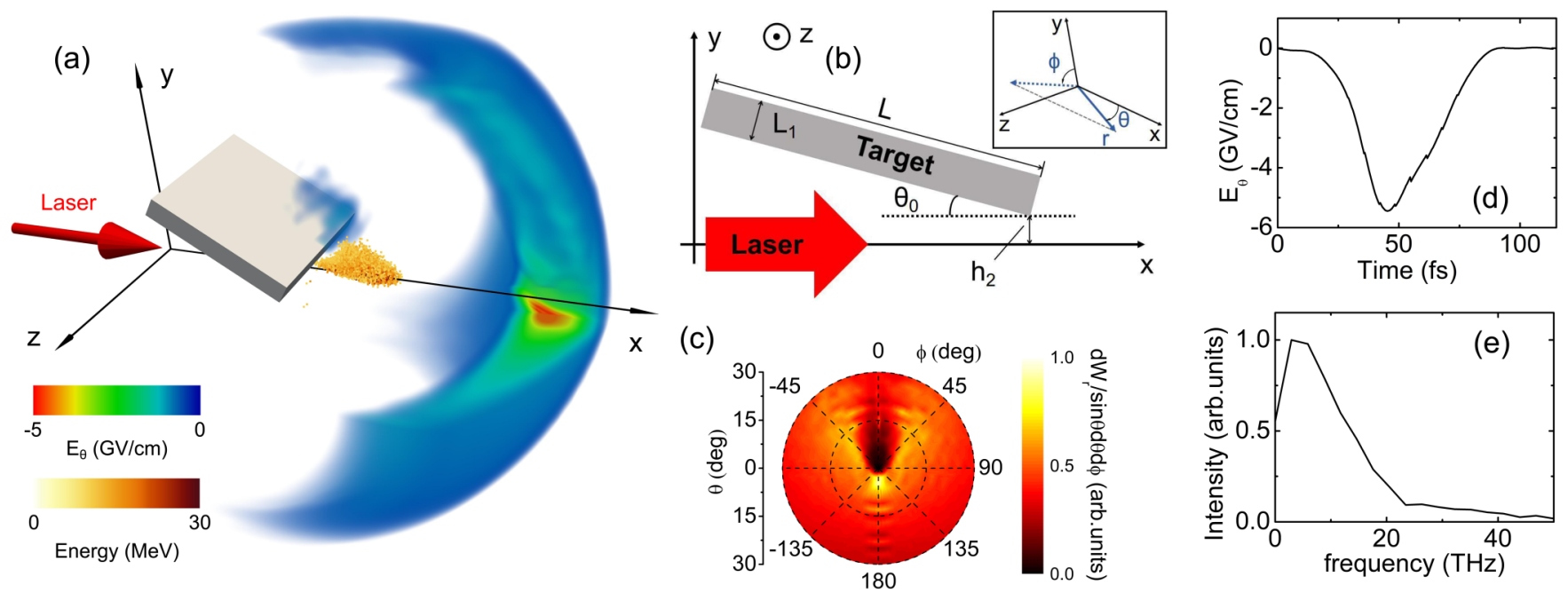

FIG. 1. (a) 3D schematic setup of the proposed scheme. The polar component of the THz electric field $E_{\theta}$ (frequency $f<60 \mathrm{THz}$ ) at simulation time $t=330 \mathrm{fs}$ is shown with rainbow color scale. Nearly a quarter of the fields $(y>-3 \mu \mathrm{m}$ and $z>0 \mu \mathrm{m})$ is removed to display the intensity inside. The orange dots are fast electrons $(\gamma>20)$ at $t=200 \mathrm{fs}$ and the color represents their energy. (b) $2 \mathrm{D}$ schematic setup of the proposed scheme view in the $x-y$ plane and demonstration of the spherical coordinate system (inset). (c) The angular distribution of radiated THz energy at $t=333$ fs. (d) $1 \mathrm{D} \mathrm{THz}$ radiation field $E_{\theta}$ observed at $\theta=-5.6^{\circ}$ and $\phi=180^{\circ}$. (e) Spectrum of the radiation field shown in (d).

\section{TERAHERTZ-PULSE GENERATION: SIMULATION}

The three-dimensional (3D) schematic setup is illustrated in Fig. 1(a) and the definitions of important parameters are shown in Fig. 1(b). A $p$-polarized incident laser pulse propagates along the $x$ axis, partially blocked by a solid foil. The front surface of the foil is tilted $\theta_{0}=30^{\circ}$ with respect to the laser axis, and it is placed such that its right edge is $x_{0}=30 \mu \mathrm{m}$ on the $x$ axis and $h_{2}=2 \mu \mathrm{m}$ above the $x$ axis.

When the laser arrives, the surface electrons are extracted and form compact beams in the laser field, which give rise to strong radiation as they pass by the right edge of the foil. The laser pulse has an intensity of $I=1.37 \times 10^{20} \mathrm{~W} / \mathrm{cm}^{2}$, which corresponds to a normalized vector potential $a_{0}=$ $e E_{0} / m_{e} c \omega_{0}=10$, where $E_{0}$ is the amplitude of laser electric field, $m_{e}$ is the mass of an electron, $c$ is the speed of light, and $\omega_{0}=2 \pi c / \lambda_{0}$ is the laser frequency, with $\lambda_{0}=1 \mu \mathrm{m}$ the wavelength. The laser beam has a Gaussian-shaped temporal profile, with FWHM duration of $T=35$ fs and a spot size of $w_{0}=4 \mu \mathrm{m}$. We have assumed the length of the foil $(L)$ is large enough so that its left edge does not touch the laser field. The thickness of the foil $\left(L_{1}\right)$ is not crucial for this study. In the simulations we present here we set $L=30 \mu \mathrm{m}$ and $L_{1}=4 \mu \mathrm{m}$. The dimension of the target in the third $(z)$ direction is $30 \mu \mathrm{m}$ in $3 \mathrm{D}$ simulations.

Due to the limitation of the computational resources, the density of the target is $15 n_{c}$ in $3 \mathrm{D}$ simulations, where $n_{c}=$ $m_{e} \omega_{0}^{2} / 4 \pi e^{2}$ is the critical density. In $2 \mathrm{D}$ simulations that will be presented later, a higher density up to $100 n_{c}$ is used. The reduced density does not have a significant effect on the main findings of this work. The particle-in-cell (PIC) simulations are carried out with the code EPOCH [32]. The dimensions of the simulation box are $x \times y \times z=100 \lambda_{0} \times 80 \lambda_{0} \times 80 \lambda_{0}$ and are sampled by $2000 \times 800 \times 800$ cells, with eight macroparticles per cell for electrons.
The laser peak reaches the right end of the target at simulation time $t=167 \mathrm{fs}$, with electron bunches [orange dots in Fig. 1(a)] enveloped in the laser field passing by the edge of the foil. The total charge is $3.2 \mathrm{nC}$. To show the properties of the CTR-like radiation producing in this process, we converted to spherical coordinates as $r=\sqrt{\left(x-x_{0}\right)^{2}+\left(y-h_{2}\right)^{2}+z^{2}}, \theta=\arccos \left[\left(x-x_{0}\right) / r\right]$, and $\phi=\arctan \left[z /\left(y-h_{2}\right)\right.$ ] [illustrated in Fig. 1(b)]. The 3D structure of the polar component of the electric fields with frequency below $60 \mathrm{THz}$ is shown by rainbow color scale in Fig. 1(a). The energy of the polar electric field $E_{\theta}$ together with the azimuthal magnetic field $B_{\phi}$ accounts for about $87 \%$ of the total radiation energy, so the $\mathrm{THz}$ emission is mainly radially polarized. The angular distribution of the $\mathrm{THz}$ energy is presented in Fig. 1(c). The $\mathrm{THz}$ emission is predominantly in the forward direction: the total $\mathrm{THz}$ energy $W_{r}$ emitted within $\theta<35^{\circ}$ is $10.7 \mathrm{~mJ}$, corresponding to a conversion efficiency of $0.83 \%$.

The temporal structure of the polar component of the electric fields observed at $\theta=5.6^{\circ}$ and $\phi=180^{\circ}$ [strongest $\mathrm{THz}$ peak in Fig. 1(c)] is shown in Fig. 1(d). The peak amplitude is $5.5 \mathrm{GV} / \mathrm{cm}$, corresponding to a normalized amplitude $a_{\mathrm{THz}}=$ 2.2, surpassing the relativistic threshold. The spectrum of radiation is shown in Fig. 1(e), from which we see that the central frequency is several THz, and over $95 \%$ of the radiated energy in the frequency domain is distributed below $20 \mathrm{THz}$.

\section{DYNAMICS OF BEAMING ELECTRONS}

The property of the electron beams is crucial for determining the energy of the CTR-like radiation; especially a high-charge beam with small divergence is favorable [20,33]. We therefore analyze the electron dynamics in the laser solid interaction, using 2D PIC simulations with higher resolutions $\left(d x \times d y=\lambda_{0} / 50 \times \lambda_{0} / 50\right)$. The laser and target parameters 

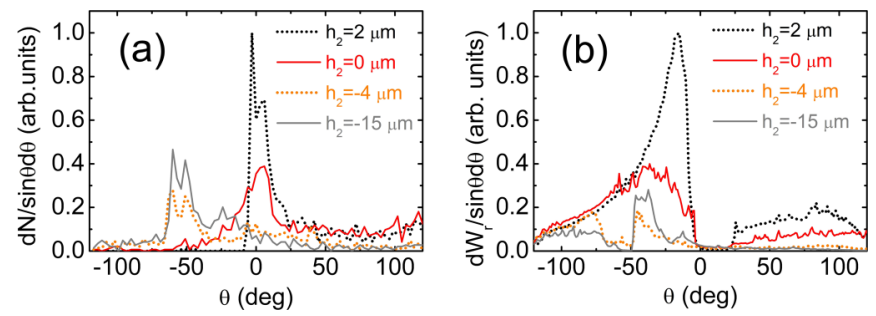

FIG. 2. (a) Angular distribution of fast electrons in the $x-y$ space at $t=333 \mathrm{fs}$. The angle $\theta$ is calculated by $\arctan [y /(x-$ $30 \mu \mathrm{m}$ )]. (b) Angular distribution of THz energy for different $h_{2}$.

are the same as in the 3D simulation expect the target density is $100 n_{c}$. Our scheme is compared with a conventional laserfoil setup, where the laser pulse specularly reflects at the front surface of the solid foil (corresponding to $h_{2} \ll-w_{0}$ in our setup).

In Figs. 2(a) and 2(b), we plot the angular distribution of the fast electrons and the corresponding $\mathrm{THz}$ radiation based on CTR, respectively. It is found that the displacement of the foil right edge and the laser axis $\left(h_{2}\right)$ has profound impact on the behavior of surface electrons. For a conventional setup $\left(h_{2}=-15 \mu \mathrm{m}\right)$, the fast electrons are broadly distributed with a small bump formed around the specular reflection direction of $\theta_{\mathrm{s}} \approx-60^{\circ}$ [34,35]. The backward CTR $\mathrm{THz}$ emission presents typical angular characteristics in this case [23]; the radiated energy is mostly distributed around $\theta_{\mathrm{s}}$, forming two small bumps (a ring shape in 3D). The radiation observed at $\theta>0^{\circ}$ is due to forward CTR when hot electrons transmit through the rear surface of the target; this has been studied by a number of experiments [17,25]. The intensity is quite low due to the small number of forward-escaping electrons. As a comparison, when $h_{2}>0$, the properties of both the electron beam and the $\mathrm{THz}$ radiation are completely different. Significant beaming is observed for the electrons emitted along the laser axis [Fig. 2(a)], which leads to a huge spike of THz energy shown in Fig. 2(b). The number of beaming electrons is 2.2 times larger than the VLA electrons at $\theta_{\mathrm{s}}$ in the case of $h_{2}=-15 \mu \mathrm{m}$.

We track the fast electrons $(\gamma>20$, chosen at $t=200 \mathrm{fs}$, when the laser peak arrives at the target) throughout the $2 \mathrm{D}$ simulations, and plot 20 representative trajectories (randomly chosen) in Figs. 3(a) and 3(b) for $h_{2}=2 \mu \mathrm{m}$ and $h_{2}=$ $-15 \mu \mathrm{m}$, respectively. In both cases, surface electrons are initially energized to relativistic energies via $J \times B$ heating and vacuum heating. Their subsequent dynamics is determined by the collective effect of the incident laser and reflected laser pulse [36-38].

The beaming effect in our scheme is attributed to VLA and the Coulomb force from the target bulk. Since the reflected fields are negligible, the electromagnetic field can be estimated by a plane wave within a few tens of microns (corresponding to the formation length of $\mathrm{THz}$ radiation). The relativistic electrons moving aligned with its wave number $\boldsymbol{k}$ can be locked in a certain phase and experience maximum acceleration and thus most likely to escape from the Coulomb barrier near the foil, as shown by red (dark gray) lines in Fig. 3(a). The electrons traveling with an angle $\left(\theta_{e}\right)$ with respect to $\boldsymbol{k}$ experience a dephasing effect; the dephasing
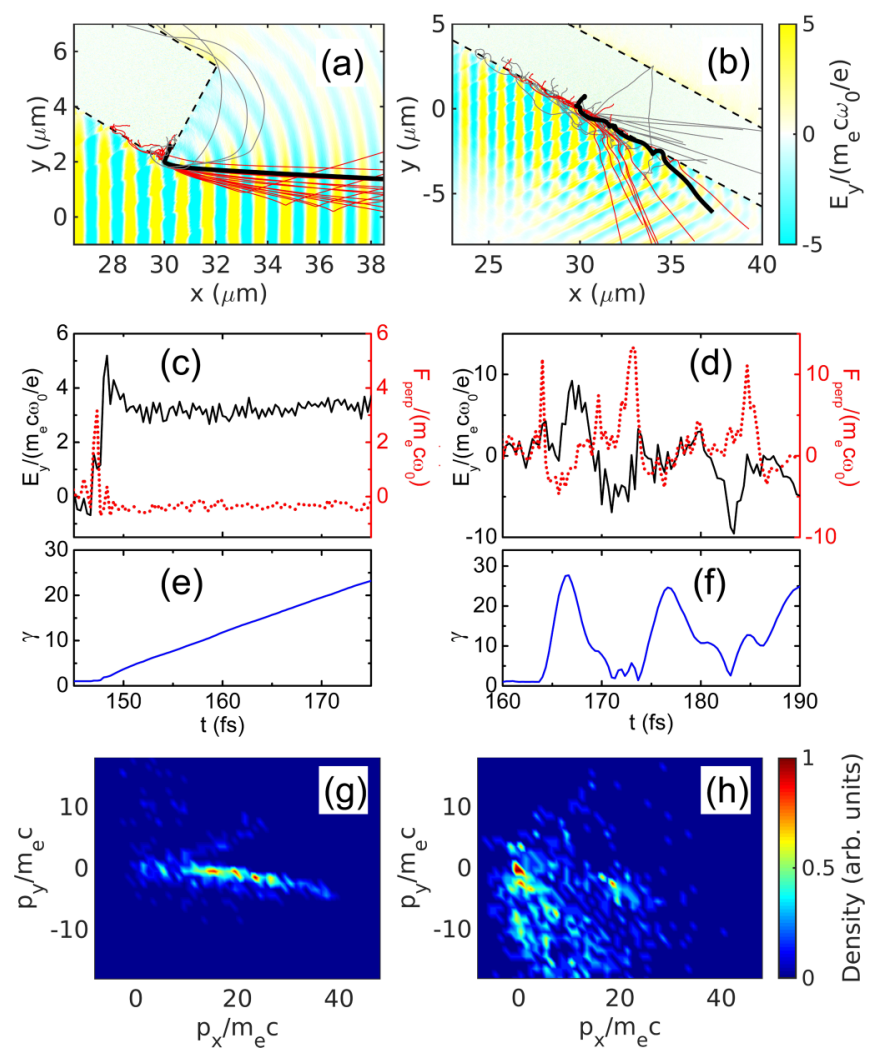

FIG. 3. (a),(b) Trajectories of 20 randomly chosen fast electrons ( $\gamma>20$ at $t=267 \mathrm{fs}$ ) are displayed, the red (dark gray) and light gray lines corresponding to electrons traveling away and towards the target bulk, respectively. The thick black lines are the representative trajectories for which (c)-(f) are plotted. On the backgrounds are snapshots of $E_{y}$ at $t=167$ fs. Dashed lines mark the initial boundaries of the target. (c),(d) Time evolution of normalized electric fields along the $y$ direction $E_{y}$ (black solid lines), as well as the Lorentz forces perpendicular to the electron velocity acting on the representative electrons (red dotted lines). (e),(f) The $\gamma$ factors versus time for the same electrons in (e) and (d). (g),(h) Electron distribution in momentum space $p_{x}-p_{y}$ at $t=175 \mathrm{fs}$. Left column is the proposed scheme $\left(h_{2}=2 \mu \mathrm{m}\right)$ and the right column shows the reference case of conventional laser-foil setup $\left(h_{2}=-15 \mu \mathrm{m}\right)$.

length can be estimated by $L_{d} \approx \lambda_{0}\left[2 / \cos \left(\theta_{e}\right)-2\right]^{-1}$. When dephasing happens, the energy of the electrons decreases $\left[d \gamma / d t=-e \boldsymbol{E} \cdot \boldsymbol{\beta} /\left(m_{e} c\right)<0\right]$ due to the inverse sign of $\boldsymbol{E}$, where $\boldsymbol{\beta}$ is the electron velocity normalized by $c$, so they are pulled back by the electrostatic fields to the target bulk [light gray curves in Fig. 3(a)]. As a result, the electrons traveling above certain critical angles are filtered by the Coulomb force. Assuming the electrons need to travel with the light for a few wavelengths to get sufficient energy, $L_{d}>\alpha \lambda_{0}$, where $\alpha$ is on the order of unity, one obtains a beam divergence $\sim 20^{\circ}$ for $\alpha \sim 5$, which agrees with the numerical results shown in Fig. 2(a).

In comparison, Fig. 3(b) shows that when the laser is totally reflected on the foil the collective effect of both incident and reflected waves leads to a complex electron motion. Figures 3(c) and 3(d) show the time evolution of electric field $E_{y}$, perpendicular force $F_{\text {perp }}$ acting on one representative electron [the trajectory is marked with black in Figs. 2(a) 

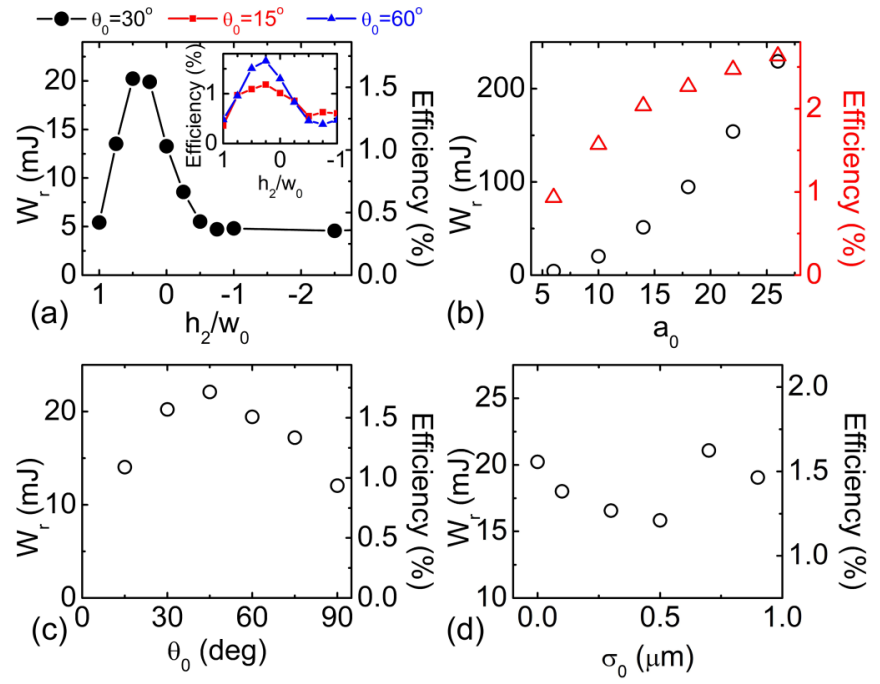

FIG. 4. THz radiation energy $W_{r}$ and conversion efficiency are plotted as functions of $h_{2}$ (a), laser amplitude $a_{0}$ (b), angle of the target relative to the laser axis $\theta_{0}$ (c), and scale length of the preplasma $\sigma_{0}$ (d). The default parameters are $h_{2}=2 \mu \mathrm{m}$ in (b),(c),(d), $a_{0}=10$ in (a), (c), (d), $\theta_{0}=30^{\circ}$ in (a),(b), (d), and $\sigma_{0}=0 \mu \mathrm{m}$ (no preplasma) in (a),(b),(c). The inset in (a) shows the conversion efficiency versus $h_{2}$ for $\theta_{0}=15^{\circ}$ and $60^{\circ}$.

and 2(b)] for both cases, whose relativistic gamma factor is shown in Figs. 3(e) and 3(f). Evidently, the changing sign of $E_{y}$ indicates the time when dephasing is happening, which is followed by a sharp reduction in the electron energy. In the meantime, the force perpendicular to its velocity $F_{\text {perp }}$ increases dramatically; this means the electron is scattered away.

In the conventional laser-foil setup, as the incident and reflected waves propagate in different directions, it is impossible for the extracted electrons to stay in phase with both; the dephasing process happens to all the electrons near the front surface, which results in a broad distribution in the momentum space map [Fig. 3(h)]. Whereas in the proposed scheme, most of the electrons in the forward-propagating beam do not experience dephasing, their energy increases monotonically and the $F_{\text {perp }}$ remains negligible. These electrons are concentrated along a thin line in the momentum space [Fig. 3(g)], indicating that they are traveling with small divergence.

\section{PARAMETRIC DEPENDENCE}

In the following, we discuss the dependence of THz energy on laser and target parameters. In Fig. 4(a), the energy of THz radiation and conversion efficiency is plotted as functions of $h_{2}$ for a fixed laser amplitude $a_{0}=10$. The optimal $h_{2}$ are $2 \mu \mathrm{m}$ and $1 \mu \mathrm{m}$, at which the strongest $\mathrm{THz}$ radiation with $W_{r}=20 \mathrm{~mJ}$ is generated, corresponding to an efficiency of $1.5 \%$. The efficiency of forward $\mathrm{THz}$ radiation within $-35^{\circ}<$ $\theta<35^{\circ}$ is about $0.9 \%$, in reasonable agreement with the $3 \mathrm{D}$ simulation result. Apparently $h_{2}$ must be smaller than the laser spot size $w_{0}$ to extract a sufficient number of electrons from the target. On the other hand, the rapid quenching around $h_{2}=0$ indicates the beaming of the electron bunch is dispersed by the reflected laser energy. It is independent of the incidence angle (as shown by the inset figure); the quenching occurs at $h_{2}=0$ for all cases, which corresponds to half of the laser energy being reflected. It can be an important signature to detect the discussed mechanism in future experiments.

We then keep $h_{2}=2 \mu \mathrm{m}$ fixed and compare the maximum $\mathrm{THz}$ energies at different initial angles of the target. For a relativistic femtosecond incident laser, fast electrons are excited most efficiently at $\theta_{0}=45^{\circ}$ [39]. This is in accordance with our simulation results presented in Fig. 4(b). The maximum $\mathrm{THz}$ energy and efficiency, appearing at $\theta_{0}=45^{\circ}$, are $22 \mathrm{~mJ}$ and $1.7 \%$, respectively. An efficiency above $1 \%$ is maintained for $15^{\circ}<\theta_{0}<75^{\circ}$.

In Fig. 4(c), the effect of laser intensity is considered. The efficiency exceeds $1 \%$ for $a_{0}=6$ and increases as the intensity grows. It is also found that, at high intensities, the efficiency saturates at about $2.5 \%$ for $a_{0}>20$. Thus the proposed scheme can maintain a high efficiency when scaling towards higher drive laser intensities.

Finally, we consider the preexpansion due to finite laser contrast by introducing a preplasma on the front surface of the target, $n(d)=100 n_{c} \exp \left(-d^{2} / \sigma_{0}^{2}\right)$. Here $d$ is the distance perpendicular to the target surface and $\sigma_{0}$ is the preplasma scale length. Figure 4(d) shows little variation of the $\mathrm{THz}$ energy with the preplasma scale length within a reasonable range; the conversion efficiency stays above $1 \%$ for all cases. It is evident that our scheme works when a preplasma exists. Note that the preexpansion leads to an effective surface location of the target, where $n\left(d_{1}\right)=n_{c}$. In this case, $h_{2}$ is measured as the distance between the right end of the effective surface and the laser axis.

\section{SUMMARY}

In conclusion, we have proposed a scheme on generating high-intensity, well-collimated $\mathrm{THz}$ radiation via the interaction between a femtosecond, relativistic incident laser pulse and a solid foil target. When the foil does not cover the laser axis and the reflected pulse is weak, a group of surface electrons are dragged out and accelerated by the laser field, along the direction of laser propagation. A substantial portion of electron energy is transferred to an intense $\mathrm{THz}$ radiation pulse when the beaming electrons pass by the target edge, due to a mechanism similar to coherent transition radiation. According to 2D and 3D PIC simulation results, the generated $\mathrm{THz}$ energy can reach $20 \mathrm{~mJ}$ and the pulse can be fully relativistic. The conversion efficiency can be over $1 \%$. Compared with traditional $\mathrm{THz}$ sources based on laser-solid interactions, the conversion efficiency is several times higher.

\section{ACKNOWLEDGMENTS}

The authors would like to thank Dr. I. Pusztai and Prof. T. Fülöp for fruitful discussions. This work is supported by the Olle Engqvist Foundation and has received funding from the European Research Council (ERC) under the European Union's Horizon 2020 research and innovation programme under Grant Agreement No. 647121. Simulations were performed on resources at Chalmers Centre for Computational Science and Engineering (C3SE) provided by the Swedish National Infrastructure for Computing (SNIC). 
[1] M. C. Hoffmann and J. A. Fülöp, J. Phys. D: Appl. Phys. 44, 083001 (2011).

[2] S. S. Dhillon, M. S. Vitiello, E. H. Linfield, A. G. Davies, M. C. Hoffmann, J. Booske, C. Paoloni, M. Gensch, P. Weightman, G. P. Williams, E. Castro-Camus, D. R. S. Cumming, F. Simoens, I. Escorcia-Carranza, J. Grant, S. Lucyszyn, M. Kuwata-Gonokami, K. Konishi, M. Koch, C. A. Schmuttenmaer, T. L. Cocker, R. Huber, A. G. Markelz, Z. D. Taylor, V. P. Wallace, J. Axel Zeitler, J. Sibik, T. M. Korter, B. Ellison, S. Rea, P. Goldsmith, K. B. Cooper, R. Appleby, D. Pardo, P. G. Huggard, V. Krozer, H. Shams, M. Fice, C. Renaud, A. Seeds, A. Stöhr, M. Naftaly, N. Ridler, R. Clarke, J. E. Cunningham, and M. B. Johnston, J. Phys. D: Appl. Phys. 50, 043001 (2017).

[3] M. Tonouchi, Nat. Photon. 1, 97 (2007).

[4] P. H. Siegel, IEEE Trans. Microwave Theory Tech. 52, 2438 (2004).

[5] E. Pickwell and V. P. Wallace, J. Phys. D: Appl. Phys. 39, R301 (2006).

[6] T. Globus, D. L. Woolard, T. Khromova, T. W. Crowe, M. Bykhovskaia, B. L. Gelmont, J. Hesler, and A. C. Samuels, J. Biol. Phys. 29, 89 (2003).

[7] J. Á. Hebling, K.-L. Yeh, M. C. Hoffmann, and K. A. Nelson, IEEE J. Sel. Top. Quantum Electron. 14, 345 (2008).

[8] T. Kampfrath, K. Tanaka, and K. A. Nelson, Nat. Photon. 7, 680 (2013).

[9] R. Matsunaga and R. Shimano, Phys. Rev. Lett. 109, 187002 (2012).

[10] H. Hirori, A. Doi, F. Blanchard, and K. Tanaka, Appl. Phys. Lett. 98, 091106 (2011).

[11] M. Shalaby and C. P. Hauri, Nat. Commun. 6, 5976 (2015).

[12] C. Vicario, M. Jazbinsek, A. V. Ovchinnikov, O. V. Chefonov, S. I. Ashitkov, M. B. Agranat, and C. P. Hauri, Opt. Express 23, 4573 (2015).

[13] D. Jang, R. M. Schwartz, D. Woodbury, J. Griff-McMahon, A. H. Younis, H. M. Milchberg, and K.-Y. Kim, Optica 6, 1338 (2019).

[14] A. D. Koulouklidis, C. Gollner, V. Shumakova, V. Y. Fedorov, A. Pugzžlys, A. Baltusžka, and S. Tzortzakis, Nat. Commun. 11, 292 (2020).

[15] A. S. Weling, B. B. Hu, N. M. Froberg, and D. H. Auston, Appl. Phys. Lett. 64, 137 (1994).

[16] Z. Wu, A. S. Fisher, J. Goodfellow, M. Fuchs, D. Daranciang, M. Hogan, H. Loos, and A. Lindenberg, Rev. Sci. Instrum. 84, 022701 (2013).

[17] G.-Q. Liao, Y. T. Li, C. Li, H. Liu, Y. H. Zhang, W. M. Jiang, X. H. Yuan, J. Nilsen, T. Ozaki, W. M. Wang, Z. M. Sheng, D. Neely, P. McKenna, and J. Zhang, Plasma Phys. Control. Fusion 59, 014039 (2016).

[18] G.-Q. Liao and Y.-T. Li, IEEE Trans. Plasma Sci. 47, 3002 (2019).

[19] W. P. Leemans, C. G. R. Geddes, J. Faure, Cs. Tóth, J. van Tilborg, C. B. Schroeder, E. Esarey, G. Fubiani, D. Auerbach, B. Marcelis, M. A. Carnahan, R. A. Kaindl, J. Byrd, and M. C. Martin, Phys. Rev. Lett. 91, 074802 (2003).
[20] C. B. Schroeder, E. Esarey, J. van Tilborg, and W. P. Leemans, Phys. Rev. E 69, 016501 (2004).

[21] C. Li, G.-Q. Liao, M.-L. Zhou, F. Du, J.-L. Ma, Y.-T. Li, W.-M. Wang, Z.-M. Sheng, L.-M. Chen, and J. Zhang, Opt. Express 24, 4010 (2016).

[22] W. J. Ding, Z. M. Sheng, and W. S. Koh, Appl. Phys. Lett. 103, 204107 (2013).

[23] W. J. Ding and Z. M. Sheng, Phys. Rev. E 93, 063204 (2016).

[24] G.-Q. Liao, Y.-T. Li, Y.-H. Zhang, H. Liu, X.-L. Ge, S. Yang, W.-Q. Wei, X.-H. Yuan, Y.-Q. Deng, B.-J. Zhu, Z. Zhang, W.-M. Wang, Z.-M. Sheng, L.-M. Chen, X. Lu, J.-L. Ma, X. Wang, and J. Zhang, Phys. Rev. Lett. 116, 205003 (2016).

[25] G.-Q. Liao, Y. Li, H. Liu, G. G. Scott, D. Neely, Y. Zhang, B. Zhu, Z. Zhang, C. Armstrong, E. Zemaityte, P. Bradford, P. G. Huggard, D. R. Rusby, P. McKenna, C. M. Brenner, N. C. Woolsey, W. Wang, Z. Sheng, and J. Zhang, Proc. Natl. Acad. Sci. USA 116, 3994 (2019).

[26] S. Li and R. R. Jones, Nat. Commun. 7, 13405 (2016).

[27] D. J. Park, B. Piglosiewicz, S. Schmidt, H. Kollmann, M. Mascheck, and C. Lienau, Phys. Rev. Lett. 109, 244803 (2012).

[28] Th. Fennel, T. Döppner, J. Passig, Ch. Schaal, J. Tiggesbäumker, and K.-H. Meiwes-Broer, Phys. Rev. Lett. 98, 143401 (2007).

[29] S. Zherebtsov, T. Fennel, J. Plenge, E. Antonsson, I. Znakovskaya, A. Wirth, O. Herrwerth, F. Süßmann, C. Peltz, I. Ahmad, S. A. Trushin, V. Pervak, S. Karsch, M. J. J. Vrakking, B. Langer, C. Graf, M. I. Stockman, F. Krausz, E. Rühl, and M. F. Kling, Nat. Phys. 7, 656 (2011).

[30] J. Passig, R. Irsig, N. X. Truong, T. Fennel, J. Tiggesbäumker, and K. H. Meiwes-Broer, New J. Phys. 14, 085020 (2012).

[31] D. E. Cardenas, T. M. Ostermayr, L. Di Lucchio, L. Hofmann, M. F. Kling, P. Gibbon, J. Schreiber, and L. Veisz, Sci. Rep. 9, 7321 (2019).

[32] T. D. Arber, K. Bennett, C. S. Brady, A. Lawrence-Douglas, M. G. Ramsay, N. J. Sircombe, P. Gillies, R. G. Evans, H. Schmitz, A. R. Bell, and C. P. Ridgers, Plasma Phys. Controlled Fusion 57, 113001 (2015).

[33] L. Yi and T. Fülöp, Phys. Rev. Lett. 123, 094801 (2019).

[34] M. Thévenet, A. Leblanc, S. Kahaly, H. Vincenti, A. Vernier, F. Quéré, and J. Faure, Nat. Phys. 12, 355 (2016).

[35] Y. Tian, J. Liu, W. Wang, C. Wang, A. Deng, C. Xia, W. Li, L. Cao, H. Lu, H. Zhang, Y. Xu, Y. Leng, R. Li, and Z. Xu, Phys. Rev. Lett. 109, 115002 (2012).

[36] N. Naumova, I. Sokolov, J. Nees, A. Maksimchuk, V. Yanovsky, and G. Mourou, Phys. Rev. Lett. 93, 195003 (2004).

[37] Y. T. Li, X. H. Yuan, M. H. Xu, Z. Y. Zheng, Z. M. Sheng, M. Chen, Y. Y. Ma, W. X. Liang, Q. Z. Yu, Y. Zhang, F. Liu, Z. H. Wang, Z. Y. Wei, W. Zhao, Z. Jin, and J. Zhang, Phys. Rev. Lett. 96, 165003 (2006).

[38] M. Chen, Z.-M. Shenga, J. Zheng, Y.-Y. Ma, M. A. Bari, Y.-T. Li, and J. Zhang, Opt. Express 14, 3093 (2006).

[39] P. Gibbon, Phys. Rev. Lett. 73, 664 (1994). 